\title{
Melatonin alleviates traumatic brain injury-induced anxiety-like behaviors in rats: Roles of the protein kinase A/cAMP-response element binding signaling pathway
}

\author{
LING-LING XIE ${ }^{1}$, SHAN-SHAN LI ${ }^{2}$, YONG-JIAN FAN ${ }^{3}$, MAN-MAN $\mathrm{QI}^{4}$ and ZHUANG-ZHUANG LI ${ }^{1}$ \\ ${ }^{1}$ Department of Pharmacy; ${ }^{2}$ Clinical Lab; Departments of ${ }^{3}$ Ultrasonography and ${ }^{4}$ Anesthesiology, \\ Cangzhou Central Hospital, Cangzhou, Hebei 061000, P.R. China
}

Received May 22, 2021; Accepted October 21, 2021

DOI: $10.3892 / \mathrm{etm} .2022 .11173$

\begin{abstract}
Melatonin is a hormone produced by the pineal gland. Given its capabilities of neuroprotection and low neurotoxicity, melatonin could be a therapeutic strategy for traumatic brain injury (TBI). The present study was conducted to determine the neuroprotective effects of melatonin on TBI-induced anxiety and the possible molecular mechanism. Rats were randomly divided into seven groups. The rodent model of TBI was established using the weight-drop method. Melatonin was administered by intraperitoneal injection at a dose of $10 \mathrm{mg} / \mathrm{kg}$ after TBI. H89 $(0.02 \mathrm{mg} / \mathrm{kg})$, a special protein kinase A (PKA) inhibitor, or dibutyryl-cyclic adenosine monophosphate (cAMP; $0.1 \mathrm{mg} / \mathrm{kg}$ ), an activator of PKA, were administered by stereotactic injection of the brain to evaluate the roles of PKA and cAMP-response element-binding protein (CREB) in melatonin-related mood regulation, respectively. At 30 days post-TBI, the changes in anxiety-like behaviors in rats were measured using the open field and elevated plus maze tests. At $24 \mathrm{~h}$ post-TBI, the number of activated astrocytes and neuronal apoptosis were evaluated using immunofluorescence assay. The expression levels of inflammatory cytokines (TNF- $\alpha$ and IL-6) in the amygdala were measured using an enzyme-linked immunosorbent assay. The expression levels of PKA, phosphorylated (p)-PKA, CREB, p-CREB, NF- $\kappa B$ and p-NF- $\kappa B$ in the amygdala were detected using western blotting. It was revealed that melatonin partially reversed TBI-induced anxiety-like behavior in rats, and decreased the number of activated astrocytes and neuronal apoptosis in the amygdala induced by TBI. H89 partially blocked the neuroprotective effects of melatonin; while dibutyryl-cAMP not only reduced the H89-induced emotional disturbance but also enhanced the
\end{abstract}

Correspondence to: Dr Zhuang-Zhuang Li, Department of Pharmacy, Cangzhou Central Hospital, 16 Xinhua West Road, Yunhe, Cangzhou, Hebei 061000, P.R. China

E-mail:1zz8700v@163.com

Key words: melatonin, traumatic brain injury, apoptosis, anxiety, protein kinase A, cAMP-response element-binding protein protective effects of melatonin against TBI. Overall, melatonin can alleviate TBI-induced anxiety-like behaviors in rats. Moreover, the underlying mechanism may be associated with the activation of the PKA/CREB signaling pathway.

\section{Introduction}

Traumatic brain injury (TBI) is one of the primary causes of disability and death in young adults and children worldwide in a 2020 study $(1,2)$. TBI not only causes major mechanical injury of cerebral tissues but also induces secondary mood changes, including anxiety, that persist for years post-injury and severely impair the quality of life for patients $(3,4)$. The amygdala is prominently involved in neurobiological models of anxiety $(5,6)$. To date, several pathological processes responsible for neuronal death, such as excitotoxicity, oxidative stress, inflammation and apoptosis, have been revealed to be involved in the secondary damage (cell death, excitotoxicity, oxidative stress and inflammation) of TBI $(7,8)$. Neuroinflammation is characterized by glial activation (such as the microglia and astrocytes) and increased release of inflammatory substances within the brain, and it is important in short- and long-term neuronal injuries post-TBI (9). However, only a few effective strategies have been proven to improve the clinical prognosis of TBI (10).

Melatonin (N-acetyl 5-methoxytryptamine), a major product of the pineal gland, has been demonstrated to generate neuroprotective effects against a series of central nervous system (CNS) disease models, including cerebral ischemia (11), brain and spinal cord trauma (12) and subarachnoid and intracerebral hemorrhage (13). It possesses fundamental characteristics such as low neurotoxicity and high permeability in the blood-brain barrier (14). Previous studies have reported that melatonin ameliorates secondary injury (for example, by decreasing apoptosis, inflammation and oxidative stress) (15-17) and also improves functional deficits (such as memory, learning and motor activities). However, to the best of our knowledge, there is no clear information on the clinical therapeutic effects of melatonin in patients with TBI. Based on the aforementioned observations, the present study hypothesized that melatonin reduces neuronal injury induced by TBI. 
The cyclic adenosine monophosphate/protein kinase A (cAMP/PKA) signaling pathway plays an important role in controlling the proliferation and differentiation of progenitor cells and the production of cytokines (18). It has been revealed that PKA phosphorylation is associated with CNS injury in vivo and in vitro (19). Furthermore, PKA plays an important role in the activation of the cAMP-response element-binding protein (CREB) (20). Activation of the PKA/CREB signaling pathway has been suggested to exert neuroprotective effects and to inhibit apoptotic cells in brain tissues (21). Therefore, regulation of amygdaloid PKA may reduce social avoidance and anxiety-like phenotypes (22). Moreover, melatonin administration ameliorates cerebral damage through the modulation of the phosphorylated (p)-AMPK/p-CREB signaling pathways and induces CREB activation correlated with long-term memory and cognition in vitro (23). p-CREB reduces neuroinflammation by regulating $\mathrm{p}-\mathrm{NF}-\kappa \mathrm{B}$ and reducing the transcription of inflammatory mediators (24). However, the roles of the PKA/CREB signaling pathway in mood changes post-TBI, including depression and anxiety, remain obscure. Moreover, whether melatonin has the potential to be developed as a therapeutic strategy against TBI mediated by the PKA/CREB signaling pathway required investigation.

The present study was conducted to investigate the effects of melatonin on anxiety-like behaviors and the pathological changes in a rodent model of TBI. The present study also examined whether the PKA/CREB signaling pathway was involved in the neuroprotective effects of melatonin.

\section{Materials and methods}

Experimental animals. Adult male Sprague-Dawley rats ( $\mathrm{n}=228$; 6-8 weeks old) weighing 300-350 g were purchased from Liaoning Changsheng Biotechnology and raised at a stable temperature $\left(25 \pm 1^{\circ} \mathrm{C}\right)$, humidity $(50 \pm 10 \%)$ in a 12 -h light/12-h dark alternating cycle with free access to food and water. The present study was approved by the Animal Review Board of Cangzhou Central Hospital [Cangzhou, China; approval no. 2020-013-02(Z)]). All animal experiments were performed according to the standards of the Institutional Animal Care and Use Committee at the Cangzhou Central Hospital. The animal health and behavior were monitored every day.

Once behavioral tests were finished, the rats were euthanized by intraperitoneal injection (i.p) of sodium pentobarbital $(200 \mathrm{mg} / \mathrm{kg})$. If rats were still alive after the experiments, euthanasia was conducted. If the rats lost their appetite for 5 days or stopped drinking for 3 days, lost $>20 \%$ of their body weight and were unable to eat and drink before the scheduled experimental endpoint, euthanasia was also conducted. Electrocardiographic monitoring (straight lines) was used to detect the death of rats.

Group assignment and drug treatment. In experiment series 1, adult male Sprague-Dawley rats were randomly divided (computer-based randomization) into one of the following four groups: i) Sham; ii) TBI; iii) TBI + Mel (melatonin); and iv) $\mathrm{TBI}+\mathrm{S}$ (saline). Melatonin $(10 \mathrm{mg} / \mathrm{kg})$ was dissolved in saline containing $2 \%$ ethanol was administered via i.p immediately after TBI (25). An equal volume of saline containing
$2 \%$ ethanol was administered through i.p immediately after $\mathrm{TBI}$ in the $\mathrm{TBI}+\mathrm{S}$ group.

In experiment series 2, adult male Sprague-Dawley rats were randomly assigned to one of the following groups: i) $\mathrm{TBI}+\mathrm{Mel}+\mathrm{S}$; ii) $\mathrm{TBI}+\mathrm{Mel}+\mathrm{H} 89$; and iii) $\mathrm{TBI}+$ Mel + dibutyryl-cAMP (dbcAMP). H-89 (0.2 mg/100 g; cat. no. HY-15979A; MedChemExpress) is a potent and selective inhibitor of PKA (26). DbcAMP (1 mg/100 g; cat. no. HY-B0764A; MedChemExpress) selectively activates cAMP-dependent protein kinase (PKA) (27). H89 and dbcAMP were dissolved in saline (containing 10\% dimethyl sulfoxide and $20 \%$ sulfobutylether $\beta$-cyclodextrin) and were applied by stereotactic injection 15 min before surgery. After anesthesia with sevoflurane (3-4\%), a hole was made with microbore dental drill, then stainless-steel guide cannulae (28-gauge) were stereotactically injected to bilaterally target the amygdala $(2.8 \mathrm{~mm}$ posterior to the bregma, $5.3 \mathrm{~mm}$ lateral from the midline and $6.25 \mathrm{~mm}$ ventral from the skull surface). To observe the interaction of H89 or dbcAMP on melatonin, the Sham, Sham + Mel + H89 and Sham + Mel + dbcAMP groups were established. Melatonin, H89, and dbcAMP were administered as aforementioned (Fig. 1). The number of animals required for each experiment in each group and the total number of animals were detailed in Table I.

TBI model. TBI was established using the weight-drop (WD) method $(9,28)$. The animals were placed on a platform covered with foam and anesthetized with sevoflurane (7-8\% for induction, and $3-4 \%$ for maintenance). A bone window with a diameter of 6-mm was drilled $(3.5 \mathrm{~mm}$ behind the bregma and $2.5 \mathrm{~mm}$ to the right of the midline). A stainless-steel planchet (diameter, 6-mm; thickness, 5-mm) was attached. An iron weight $(20 \mathrm{~g})$ was freely dropped from a height of $25 \mathrm{~cm}$, and the dura was kept intact. In sham-operated animals, the skull was exposed under anesthesia and skin incisions were then closed using silk sutures without TBI. Rats in the TBI or Sham group were treated by another experimenter blinded to behavioral tests.

Open field test (OFT). The OFT was performed to evaluate anxiety and inquiry behavior (29). The site was a large open field $(60 \times 60 \times 40 \mathrm{~cm})$, which was segmented into 16 equal squares. The test was conducted at 30 days post-TBI. The site was cleaned with $75 \%$ alcohol to prevent foreign smells that may influence behavior. The rat was positioned in the site, and the distance, speed, the number of squares crossed (with four paws), the time spent in the center and the rearing times were recorded for $90 \mathrm{sec}$. The trajectory images were analyzed using a computerized tracking system (supermaze XR-Xmaze; Shanghai XinRuan Information Technology Co. Ltd.

Elevated plus maze (EPM). The EPM consisted of two closed arms $(50 \mathrm{~cm} \mathrm{~L} \mathrm{x} 10 \mathrm{~cm} \mathrm{~W} \mathrm{x} 40 \mathrm{~cm} \mathrm{H})$ and two open arms with the same size at $90^{\circ}$ angles to each other. The maze was $70-\mathrm{cm}$ high from the floor. At 30 days post-TBI the rat was placed in the center zone of the maze with its nose directed toward the same open arm and allowed to explore the maze for $5 \mathrm{~min}$. The maze was cleaned with $75 \%$ ethanol after each test. The time spent on the open and closed arms, the distance traveled and the number of entries were recorded and 


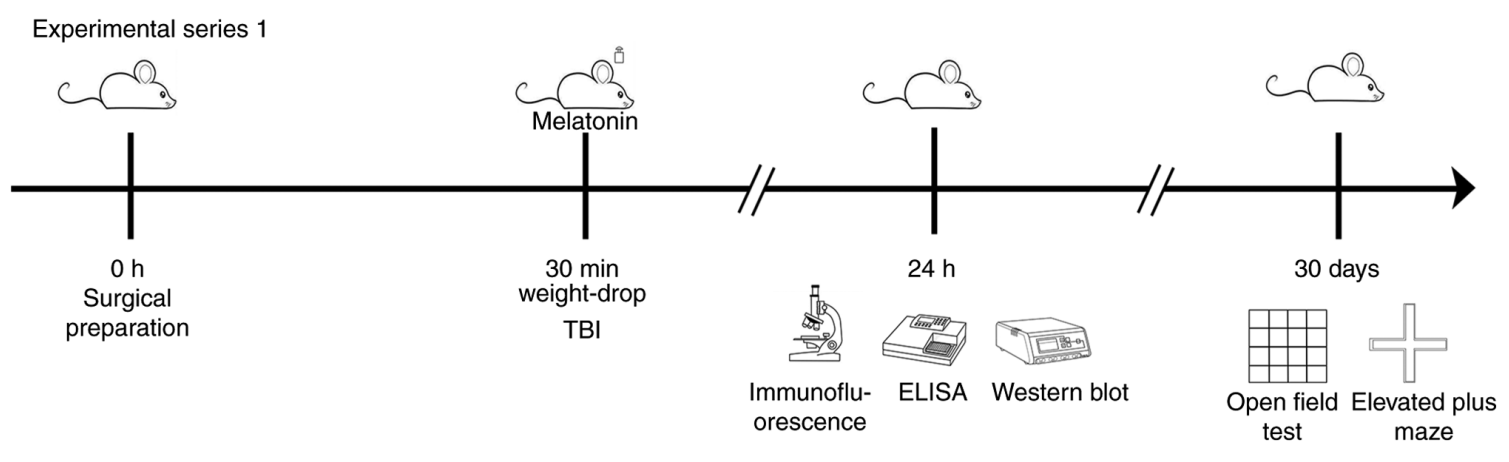

Experimental series 2

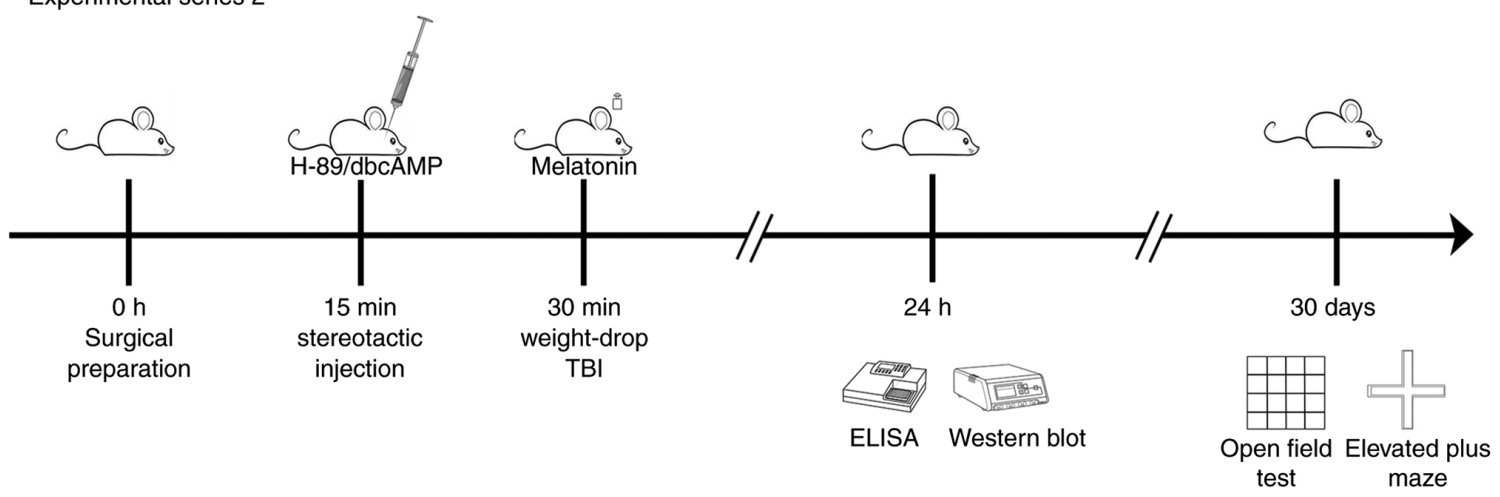

Figure 1. Schematic of experiments on rats. Rats were subjected to TBI, ELISA, immunofluorescence staining, western blotting analysis, elevated plus maze and an open field test were evaluated. TBI, traumatic brain injury; ELISA, enzyme-linked immunosorbent assay.

analyzed using the software (XR-XG2011; Shanghai XinRuan Information Technology Co. Ltd.). An entry was calculated when all four paws were entered into an arm. The anxiety level was evaluated by counting the percentage of open arm entries (number of open arm entries/number of all arm entries) and the percentage of open arm time (time spent in the open arms/total time spent in all arms). The locomotor activity was evaluated using the total moving distance in the maze. Once behavioral tests (OFT and EPM) were finished, euthanasia was conducted by intraperitoneal injection of sodium pentobarbital (200 mg/kg).

Immunofluorescence. At $24 \mathrm{~h}$ after TBI, rats were anaesthetized with sevoflurane (8\%) and transcardially perfused with cold PBS followed by $4 \%$ formaldehyde. The cerebral tissues containing the amygdala were fixed in $10 \%$ neutral-buffered formalin at $25^{\circ} \mathrm{C}$ for $24 \mathrm{~h}$, embedded in solid paraffin and cut into a thickness of $4-\mu \mathrm{m}(\mathrm{n}=6)$. The paraffin sections were dewaxed, washed with ethanol for $2 \mathrm{~min}$ at $25^{\circ} \mathrm{C}$ and hydrated (100\% ethanol for $3 \mathrm{~min}, 95 \%$ ethanol for $2 \mathrm{~min}, 80 \%$ ethanol for $2 \mathrm{~min}, 75 \%$ ethanol for $2 \mathrm{~min}, \mathrm{H}_{2} \mathrm{O}$ for $1 \mathrm{~min}$ ) at room temperature, blocked using QuickBlock ${ }^{\mathrm{TM}}$ Blocking Buffer (P0222; Beyotime Biotechnology) for $1 \mathrm{~h}$ at room temperature and then incubated overnight at $4^{\circ} \mathrm{C}$ with the primary mouse antibody against glial fibrillary acidic protein antibody (GFAP; 1:100; cat. no. ab4648; Abcam). On the next day, the pathological sections were washed three times in phosphate buffer saline (PBS) and incubated with the corresponding secondary antibodies (Cy3-conjugated goat anti-mouse IgG; 1:500; cat. no. A0521; Beyotime Institute of Biotechnology) for $1 \mathrm{~h}$ at $25^{\circ} \mathrm{C}$ in the dark.
Neuronal apoptosis was detected using TUNEL staining. After dewaxing and hydration as aforementioned), the slices were washed three times in PBS before adding $20 \mu \mathrm{g} / \mathrm{ml}$ of Proteinase $\mathrm{K}$ (cat. no. st533; Beyotime Institute of Biotechnology) at $37^{\circ} \mathrm{C}$ for $35 \mathrm{~min}$. The slices were then incubated overnight at $4^{\circ} \mathrm{C}$ with the primary polyclonal mouse antibody against neuronal nuclei (NeuN, 1:200, ab104224; Abcam). After washing with PBS, the sections were incubated with TDT enzyme including a fluorescent labeling solution [One-step TUNEL cell apoptosis detection kit (fluorescent green); cat. no. C1088; Beyotime Institute of Biotechnology] at $37^{\circ} \mathrm{C}$ for $60 \mathrm{~min}$ in the dark. Subsequently, $5 \mu \mathrm{g} / \mathrm{ml}$ of Antifade Mounting Medium with 4',6-diamidino-2-phenylindole (DAPI; cat. no. P0131; Beyotime Institute of Biotechnology) was used for $3 \mathrm{~min}$ at $25^{\circ} \mathrm{C}$ to label the cell nuclei locations. Immunofluorescence images were captured using a fluorescence microscope (Olympus Corporation). A total of 6 fields of view were evaluated in each tissue section (x400 magnification). The position of amygdala was defined according to the corresponding brain anatomical map stained with DAPI (Fig. S2), which was $\sim 5.3 \mathrm{~mm}$ from the midline and $6.25 \mathrm{~mm}$ from the parietal cortex. The number of GFAP-positive cells, the number of activated astrocytes and the percentage of NeuNand TUNEL-positive cells were calculated by Image-pro plus 6.0 software (Media Cybernetics, Inc.).

Western blotting. At $24 \mathrm{~h}$ post-TBI, rats were anaesthetized with sevoflurane (8\%) and transcardially perfused with cold PBS, total protein was extracted from the amygdala tissues and lysed in lysis buffer (cat. no. P0013; Beyotime Institute of Biotechnology). Subsequently, $30 \mu \mathrm{g}$ protein per lane was electrophoresed on $12 \%$ SDS-PAGE, and the separated 
Table I. Number of rats in each experimental phase and experimental group.

A, Experimental series $1(n=120)$

\begin{tabular}{lcccc} 
Experimental group & OFT + EPM & IF & ELISA & $\begin{array}{c}\text { Western } \\
\text { blotting }\end{array}$ \\
\hline Sham $(\mathrm{n}=30)$ & 12 & 6 & 6 & 6 \\
TBI $(\mathrm{n}=30)$ & 12 & 6 & 6 & 6 \\
TBI + Mel $(\mathrm{n}=30)$ & 12 & 6 & 6 & 6 \\
TBI + S $(\mathrm{n}=30)$ & 12 & 6 & 6 & 6 \\
\hline
\end{tabular}

B, Experimental series $2(n=108)$

\begin{tabular}{|c|c|c|c|c|}
\hline Experimental group & $\mathrm{OFT}+\mathrm{EPM}$ & IF & ELISA & blottin \\
\hline $\mathrm{TBI}+\mathrm{Mel}+\mathrm{S}(\mathrm{n}=24)$ & 12 & - & 6 & 6 \\
\hline TBI + Mel + H89 (n=24) & 12 & - & 6 & 6 \\
\hline TBI + Mel + dbcAMP $(n=24)$ & 12 & - & 6 & 6 \\
\hline Sham $(n=12)$ & 12 & - & - & - \\
\hline Sham + Mel + H89 (n=12) & 12 & - & - & - \\
\hline Sham + Mel + dbcAMP $(n=12)$ & 12 & - & - & - \\
\hline
\end{tabular}

OFT, Open field test; EPM, elevated plus maze; TBI, traumatic brain injury; Mel, melatonin; S, saline; dbcAMP, dibutyryl-cAMP; IF, immunofluorescence.

proteins were transferred onto polyvinylidene fluoride membranes and blocked in Quickblock ${ }^{\mathrm{TM}}$ sealing fluid (cat. no. P0252; Beyotime Institute of Biotechnology) at $25^{\circ} \mathrm{C}$ for $10 \mathrm{~min}$, followed by incubation overnight at $4^{\circ} \mathrm{C}$ with the indicated primary antibodies as follows: P-CREB (1:500; cat. no. AF5785), CREB (1:500; cat. no. AF1018), p-PKA (1:500; cat. no. AF1942), p-NF-кB (1:500; cat. no. AF5878), NF- $\kappa$ B (1:500; cat. no. AF7569) (all Beyotime Institute of Biotechnology) and GAPDH (1:2,000; cat. no. K106389P; Beijing Solarbio Science \& Technology Co., Ltd.). The next day, the membranes were incubated with horseradish peroxidase AffiniPure goat anti-rabbit IgG $(\mathrm{H}+\mathrm{L} ; 1$ 1:1,000; cat. no. A0208; Beyotime Institute of Biotechnology) at $37^{\circ} \mathrm{C}$ for $1 \mathrm{~h}$. Anti-GAPDH Polyclonal Antibody $(1: 2,000$; cat. no. K106389P; Beijing Solarbio Science \& Technology Co., Ltd.) was used as an internal reference. The polyvinylidene fluoride membranes were incubated with ECL reagent (cat. no. P0018FM; Beyotime Institute of Biotechnology) for $5 \mathrm{~min}$ at room temperature, and protein bands were detected by a western blot detection system (Gel Doc XRS; Bio-Rad Laboratories, Inc.). The gray value of the protein bands was analyzed using the Image-pro plus 6.0 software (Media Cybernetics, Inc.).

Enzyme-linked immunosorbent assay (ELISA). At $24 \mathrm{~h}$ post-TBI, the rats were euthanized using $200 \mathrm{mg} / \mathrm{kg}$ sodium pentobarbital (i.p.) and the amygdala was separated. The TNF- $\alpha$ Assay (cat. no. PT516) and IL-6 Assay (cat. no. PI328) ELISA kits (both from Beyotime Institute of Biotechnology) were used to measure the levels of TNF- $\alpha$ and IL- 6 in the amygdala according to the manufacturer's instructions.
Statistical analysis. All statistical analyses were conducted using SPSS Statistics (version 20.0; IBM Corp.). Data are presented as mean \pm standard deviation $(n=12$ rats/group in behavioral test, $n=6$ rats/group in molecular and pathological tests). Levene's test was used to test the homogeneity of variance hypothesis. When identifying data heteroscedasticity, the logarithmic transformation was performed and the heteroscedasticity was corrected. The statistical significance between groups was evaluated using one-way analysis of variance, followed by Tukey's post hoc test. $\mathrm{P}<0.05$ was considered to indicate a statistically significant difference.

\section{Results}

Melatonin alleviates anxiety-like behaviors post-TBI. Behavioral tests (OFT and EPM) were performed to evaluate the effects of melatonin on anxiety-related behaviors. The behavioral assessments of all rats at 30 days after TBI are presented in Fig. 2. First, the results of OFT indicated that rats in all the TBI groups exhibited a decreased amount of movement; namely, a significantly shorter total distance, slower average speed, less rearing, fewer number of squares crossed and less time spent in the center compared with those in the Sham group $(\mathrm{P}<0.0001$ vs. Sham; Fig. 2A-F). However, melatonin administration after TBI exposure resulted in significantly increased moving distance, greater average speed, more time spent in the center, more squares crossed and increased rearing $(\mathrm{P}<0.0001$ vs. TBI; Fig. 2A-F). No significant differences were observed in the total moving distance, average speed, rearing, number of squares crossed and time spent in the center between rats in the TBI and TBI $+\mathrm{S}$ groups. 
A

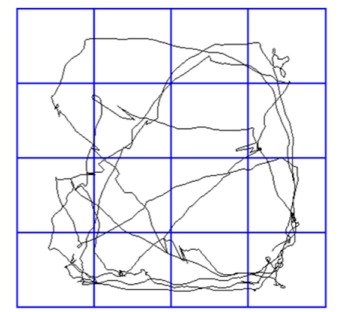

Sham

B

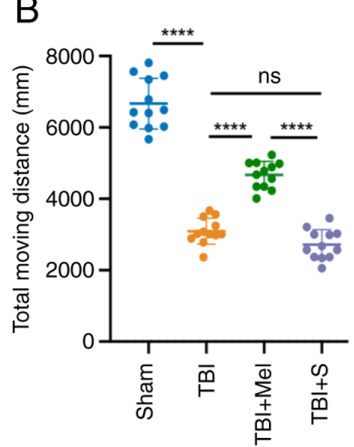

F

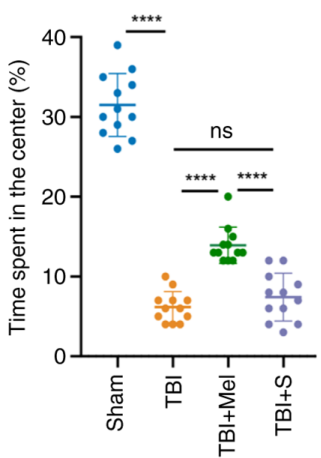

I

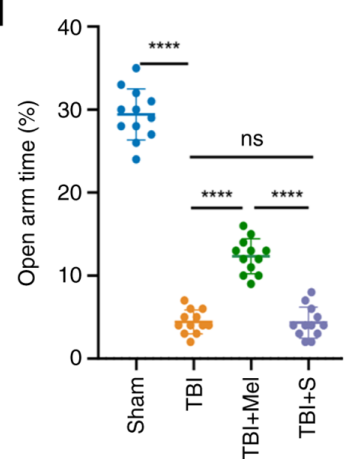

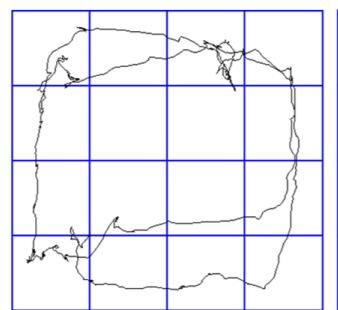

TBI

C

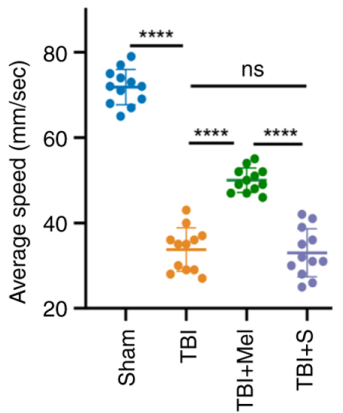

G
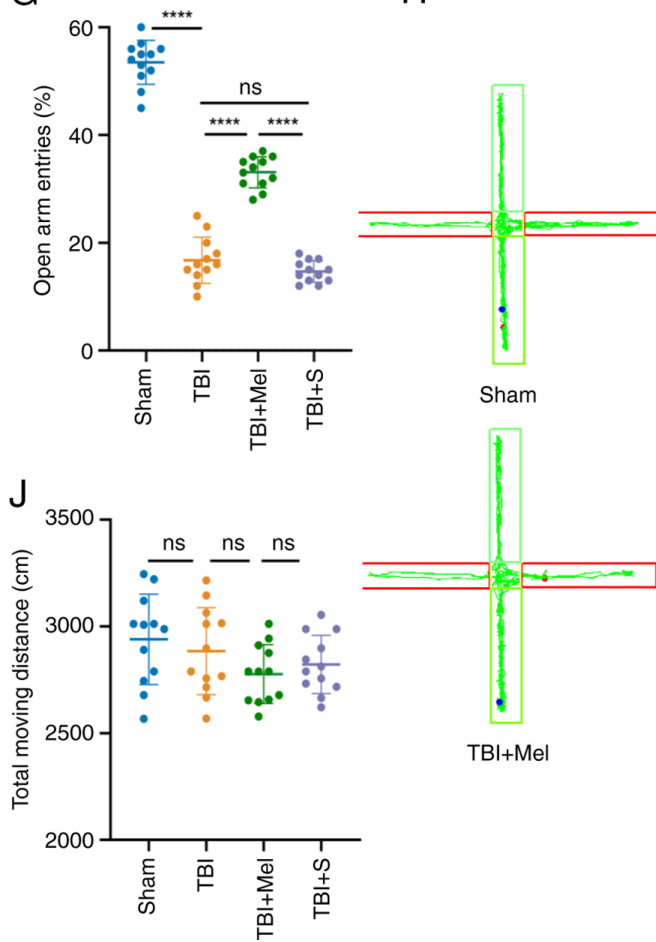

D

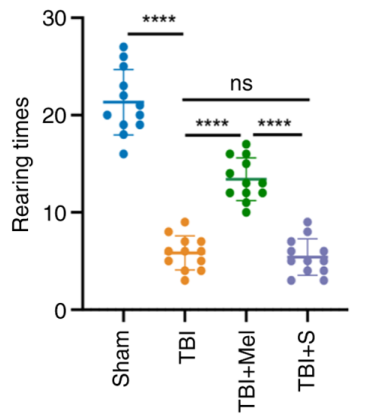

E

$\mathrm{H}$

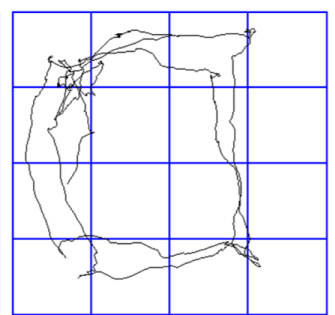

$\mathrm{TBI}+\mathrm{S}$
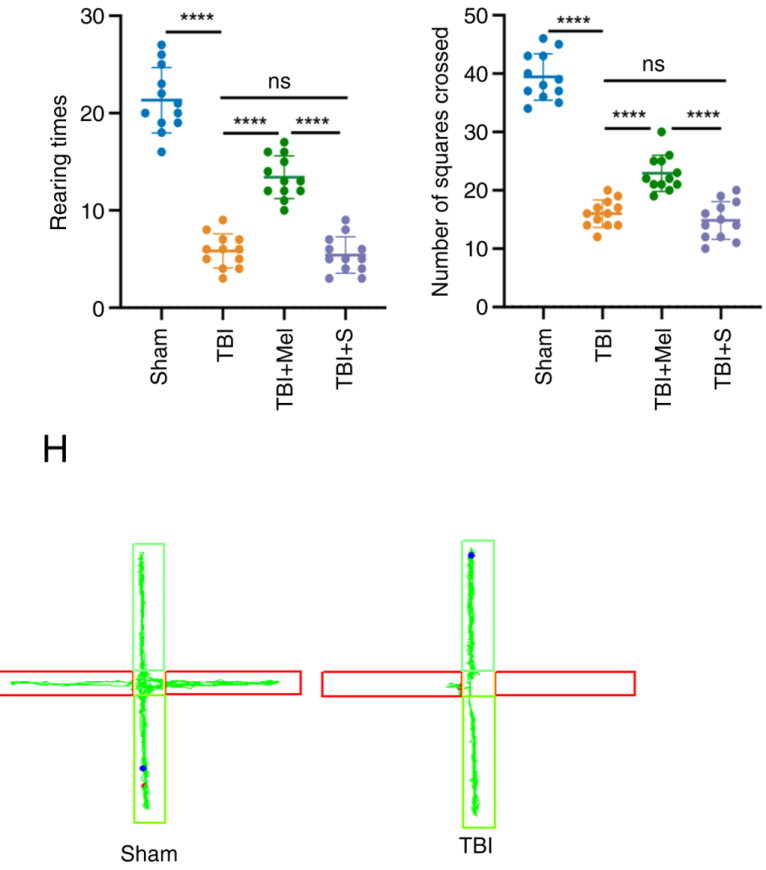

Figure 2. Melatonin alleviates anxiety-like behaviors post-TBI. Open field test at 30 days post-TBI of the experimental rats. (A) Trajectory map. (B) Total moving distance. (C) Average speed (D) Rearing times. (E) Number of squares crossed. (F) Percentage of time spent in the center. (G) Percentage of open arm entries (\%). (H) Elevated plus maze at 30 days post-TBI of the experimental rats. (I) Percentage of time spent in open arms (\%). (J) Total moving distance in elevated plus maze. $\mathrm{n}=12$ /group. ${ }^{* * * *} \mathrm{P}<0.0001$. TBI, traumatic brain injury; Mel, melatonin; $\mathrm{S}$, saline; ns, not significant.

Next, the results of EPM indicated that rats in the TBI group exhibited a significant decrease in the percentage of open arm entries and the percentage of open arm time compared with rats in the Sham group ( $\mathrm{P}<0.0001$ vs. Sham; Fig. 2G-I). Melatonin could significantly increase the percentage of open arm entries and the percentage of open arm times $(\mathrm{P}<0.0001$ vs. TBI; Fig. 2G-I). There were no significant differences in the total moving distance among the groups (Fig. 2J), which suggested that the locomotor activity of rats among the different groups in this test was not altered.
Melatonin reduces neuronal apoptosis in the region of amygdala post-TBI. At $24 \mathrm{~h}$ post-TBI, neuronal apoptosis was further evaluated by TUNEL staining combined with NeuN staining. As presented in Fig. 3A and B, there were few TUNEL-positive cells in the Sham group. Compared with the Sham group, TBI caused a significant increase in the number of TUNEL- and NeuN-positive cells ( $\mathrm{P}<0.0001$ vs. Sham group; Fig. $3 \mathrm{~A}$ and B). However, melatonin administration significantly reduced neuronal apoptosis in the amygdala and provided neuroprotective effects after TBI ( $\mathrm{P}<0.0001$ vs. TBI group; Fig. 3A and B). 

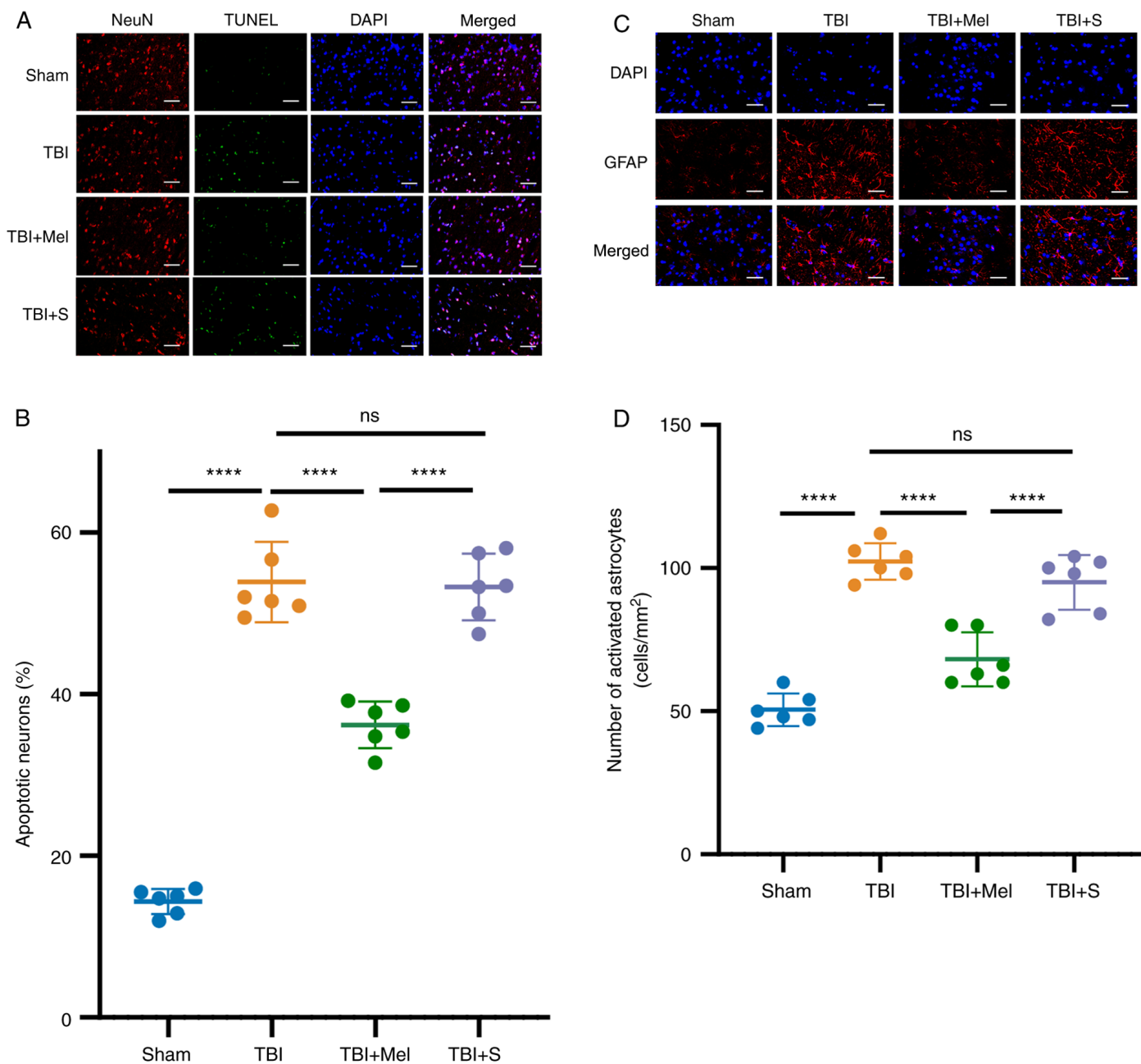

Figure 3. Melatonin reduces neuronal apoptosis and upregulates the activation of astrocytes in the region of amygdala post-TBI. (A) Representative images of NeuN (specificity marker of neurons) plus TUNEL staining in the amygdala (NeuN, red; TUNEL, green; DAPI, blue). Magnification, $\mathrm{x} 400$; scale bar=50 $\mu \mathrm{m}$. (B) Apoptosis was quantitatively observed by TUNEL staining $(\mathrm{n}=6)$. (C) Representative images of GFAP (specificity marker of astrocyte activation) in the amygdala (GFAP, red; DAPI, blue). Magnification, $\mathrm{x} 400$; scale bar=50 $\mu \mathrm{m}$. (D) Quantification of changes in the number of activated astrocytes. $\mathrm{n}=6$ rats per group. ${ }^{* * * * *} \mathrm{P}<0.0001$. TBI, traumatic brain injury; Mel, melatonin; S, saline; ns, not significant; NeuN, neuronal nuclei; DAPI, 4',6-diamidino-2-phenylindole; GFAP, glial fibrillary acidic protein.

In addition, there were no statistical differences in neuronal apoptosis between the TBI and TBI $+\mathrm{S}$ groups.

Melatonin upregulates the activation of astrocytes in the region of amygdala post-TBI. GFAP is a marker of mature and activated astrocytes. The results demonstrated that the number of GFAP-positive cells was significantly elevated at $24 \mathrm{~h}$ post-TBI compared with that in the Sham group $(\mathrm{P}<0.0001$ vs. Sham, Fig. 3C and D). However, the number of GFAP-positive cells significantly decreased in the TBI + Mel group ( $\mathrm{P}<0.001$ vs. TBI; Fig. $3 \mathrm{C}$ and $\mathrm{D})$. Moreover, no statistical difference was observed in the number of GFAP-positive cells in the region of amygdala between rats in the TBI and $\mathrm{TBI}+\mathrm{S}$ groups.

Melatonin reduces the level of inflammatory cytokines and activates the PKA/CREB signaling pathway in the amygdala post-TBI. The effects of melatonin on inflammatory cytokine levels were evaluated by ELISA. The results indicated that the levels of TNF- $\alpha$ and IL- 6 in the TBI group were significantly increased compared with those in the Sham group $(\mathrm{P}<0.0001$ vs. Sham; Fig. 4A and B). Melatonin administration significantly downregulated the expression levels of TNF- $\alpha$ and IL-6 compared with those in the TBI group ( $\mathrm{P}<0.01 \mathrm{vs}$. TBI; Fig. 4A and B).

To further understand the mechanism of action of melatonin on anxiety-like behaviors, the protein expression levels of PKA, CREB and NF- $\kappa B$ and the phosphorylation levels of the three proteins in the amygdala tissues were detected using western blotting. The expression levels of p-PKA, p-CREB and p-NF- $\kappa B$ significantly increased in the TBI groups compared with those in the Sham groups $(\mathrm{P}<0.001$ vs. Sham; Fig. $4 \mathrm{C}-\mathrm{F})$. The $\mathrm{p}$-PKA/t-PKA ratio and the $\mathrm{p}-\mathrm{CREB} / \mathrm{t}-\mathrm{CREB}$ ratio were also significantly higher in the TBI + Mel group compared with the TBI group ( $\mathrm{P}<0.01$ vs. Sham; Fig. 4C-E). In addition, the expression of $\mathrm{p}-\mathrm{NF}-\kappa \mathrm{B} / \mathrm{t}-\mathrm{NF}-\kappa \mathrm{B}$ ratio was significantly decreased in the $\mathrm{TBI}+\mathrm{Mel}$ group compared with that in the TBI group $(\mathrm{P}<0.0001$ vs. Sham; Fig. 4C and F). There was no difference between the $\mathrm{TBI}$ and $\mathrm{TBI}+\mathrm{S}$ groups in the expression of each protein.

Melatonin may alleviate TBI-induced anxiety-like behaviors through the PKA/CREB signaling pathway. Whether the exact 

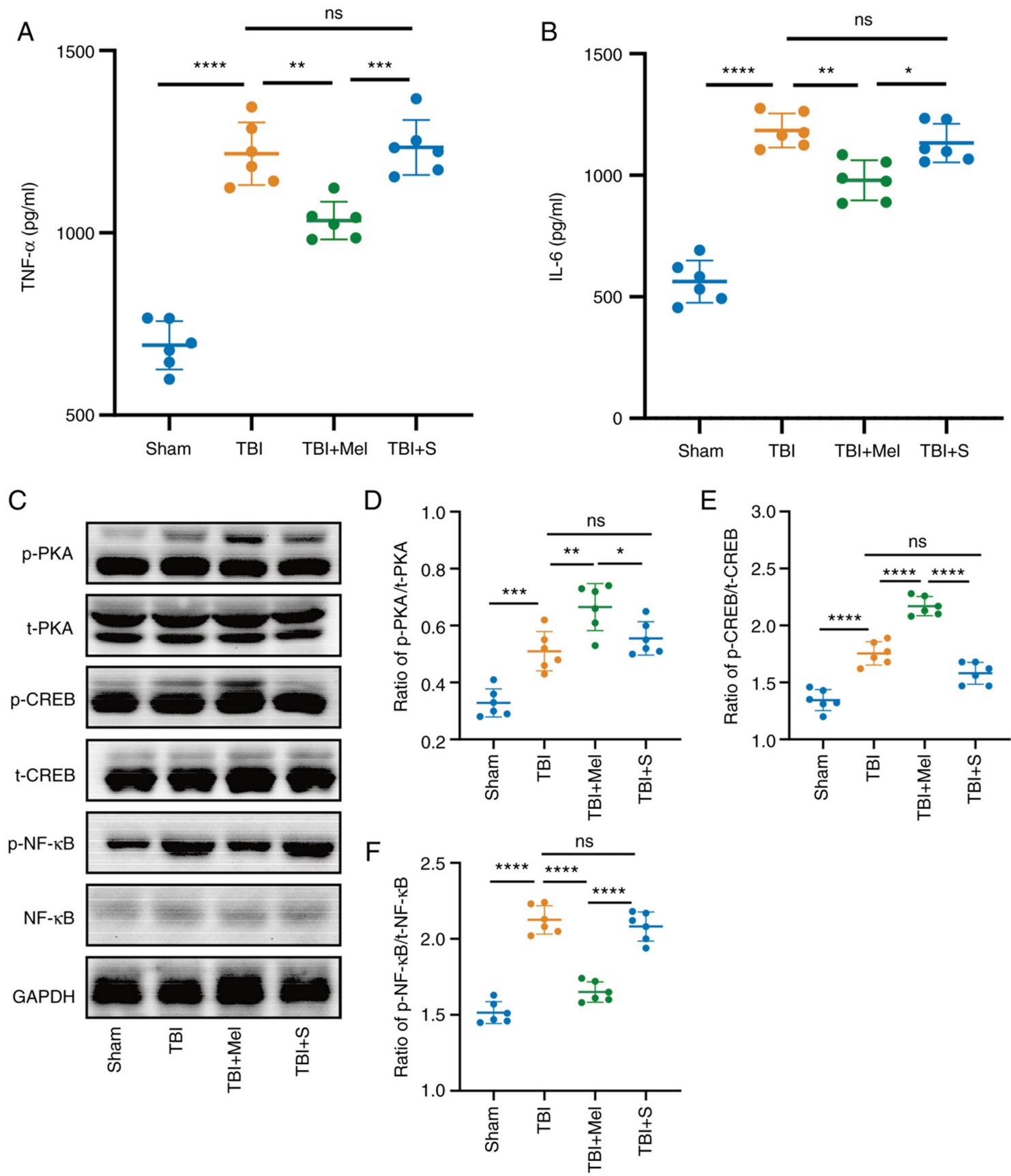

Figure 4. Melatonin reduces the level of inflammatory cytokines and activates the PKA/CREB signaling pathway in the amygdala post-TBI. (A) Expression levels of TNF- $\alpha$ in the amygdala of rats. (B) Expression levels of IL-6 in the amygdala. (C) Representative results of immunoblotting of PKA, p-PKA, CREB,

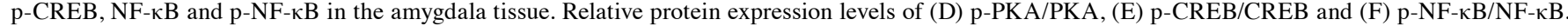
$\mathrm{n}=6$ rats per group. ${ }^{*} \mathrm{P}<0.05,{ }^{* *} \mathrm{P}<0.01,{ }^{* * *} \mathrm{P}<0.001,{ }^{* * * * *} \mathrm{P}<0.0001$. TBI, traumatic brain injury; Mel, melatonin; S, saline; ns, not significant; PKA, protein kinase A; CREB, cAMP-response element-binding protein; p-, phosphorylated; t-, total.

mechanism of action of melatonin against anxiety-like behaviors post-TBI was associated with the PKA/CREB pathway was also determined. H89 and dbcAMP were administered 15 min before TBI exposure. There was no difference among the Sham, Sham + Mel + H89 and Sham + Mel + dbcAMP groups in terms of open field and elevated maze tests (Fig. S1). Therefore, it was hypothesized that there was no interaction of $\mathrm{H} 89$ or dbcAMP with melatonin, and the three groups were eliminated in following experiment. There was a significant decrease in total moving distance, average speed, time spent in the center, number of squares crossed and rearing times in the OFT in the rats of the TBI $+\mathrm{Mel}+\mathrm{H} 89$ group $(\mathrm{P}<0.0001$ vs. TBI + $\mathrm{Mel}+\mathrm{S}$; Fig. 5A-F). The EPM results revealed that the rats in the TBI + Mel + H89 group exhibited a significant decrease in the percentage of open arm entries and the percentage of open arm times $(\mathrm{P}<0.0001$ vs. TBI $+\mathrm{Mel}+\mathrm{S}$; Fig. $5 \mathrm{G}-\mathrm{I})$. However, after the activation of PKA with dbcAMP, the protective effect of melatonin on the nervous system was strengthened and exhibited the opposite effect of H89 application.

The effects of melatonin on inflammatory cytokine levels in the amygdala were measured by ELISA. Results revealed that the levels of TNF- $\alpha$ and IL- 6 in the TBI + Mel + H89 

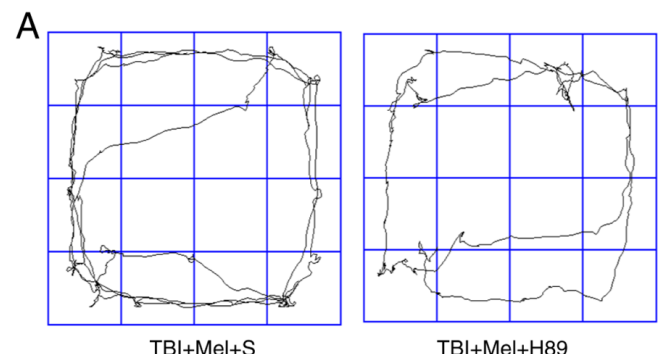

$\mathrm{TBI}+\mathrm{Mel}+\mathrm{H} 89$

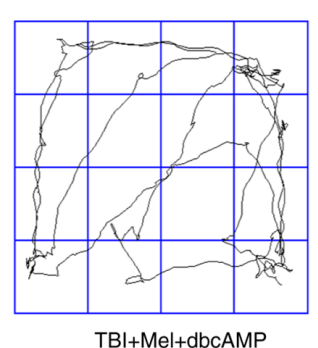

$\mathrm{TBI}+\mathrm{Mel}+\mathrm{dbcAMP}$

$\mathrm{D}$
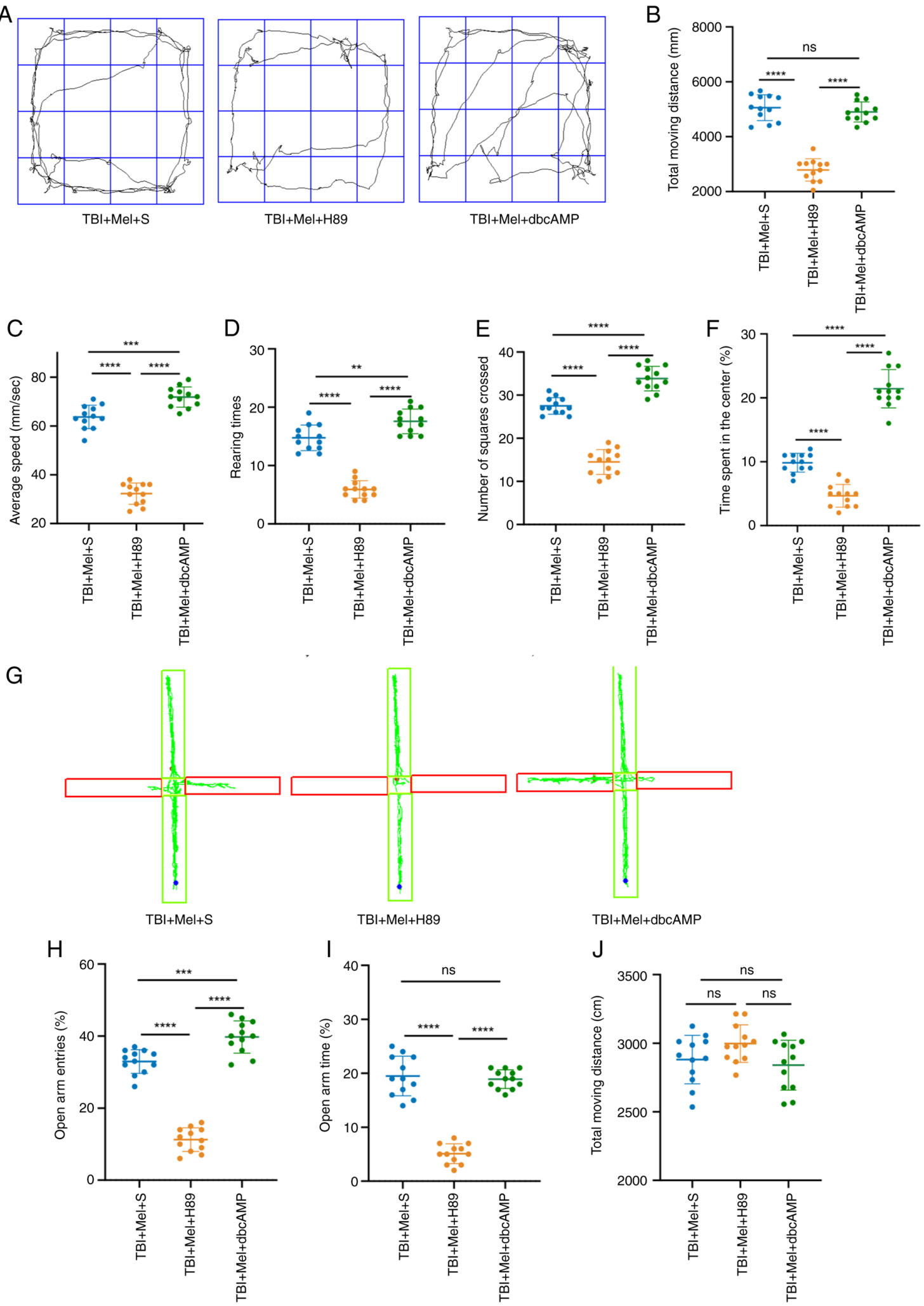

Figure 5. Effects of the administration of H89 and dbcAMP on the anxiety-like behaviors of melatonin-treated TBI rats. OFT was performed at 30 days post-TBI of the experimental rats. (A) Trajectory map. (B) Total moving distance. (C) Average speed. (D) Rearing times. (E) Number of squares crossed. (F) Percentage of time spent in the center. (G) Elevated plus maze at 30 days post-TBI of the experimental rats. (H) Percentage of open arm entries (\%). (I) Percentage of time spent in open arms (\%). (J) Total moving distance in EPM. $\mathrm{n}=12$ rats per group. ${ }^{* *} \mathrm{P}<0.01,{ }^{* * * *} \mathrm{P}<0.001,{ }^{* * * * *} \mathrm{P}<0.0001$. TBI, traumatic brain injury; Mel, melatonin; S, saline; ns, not significant; dbcAMP, dibutyryl-cAMP.

group were significantly increased compared with those in the $\mathrm{TBI}+\mathrm{Mel}+\mathrm{S}$ group $(\mathrm{P}<0.05$ vs. $\mathrm{TBI}+\mathrm{Mel}+\mathrm{S}$; Fig. 6$)$. DbcAMP application (TBI + Mel + dbcAMP) significantly downregulated the expression levels of inflammatory cytokines compared with those in the $\mathrm{TBI}+\mathrm{Mel}+\mathrm{S}(\mathrm{P}<0.05$; Fig. 6) and TBI + Mel + H89 groups (P<0.0001; Fig. 6). 

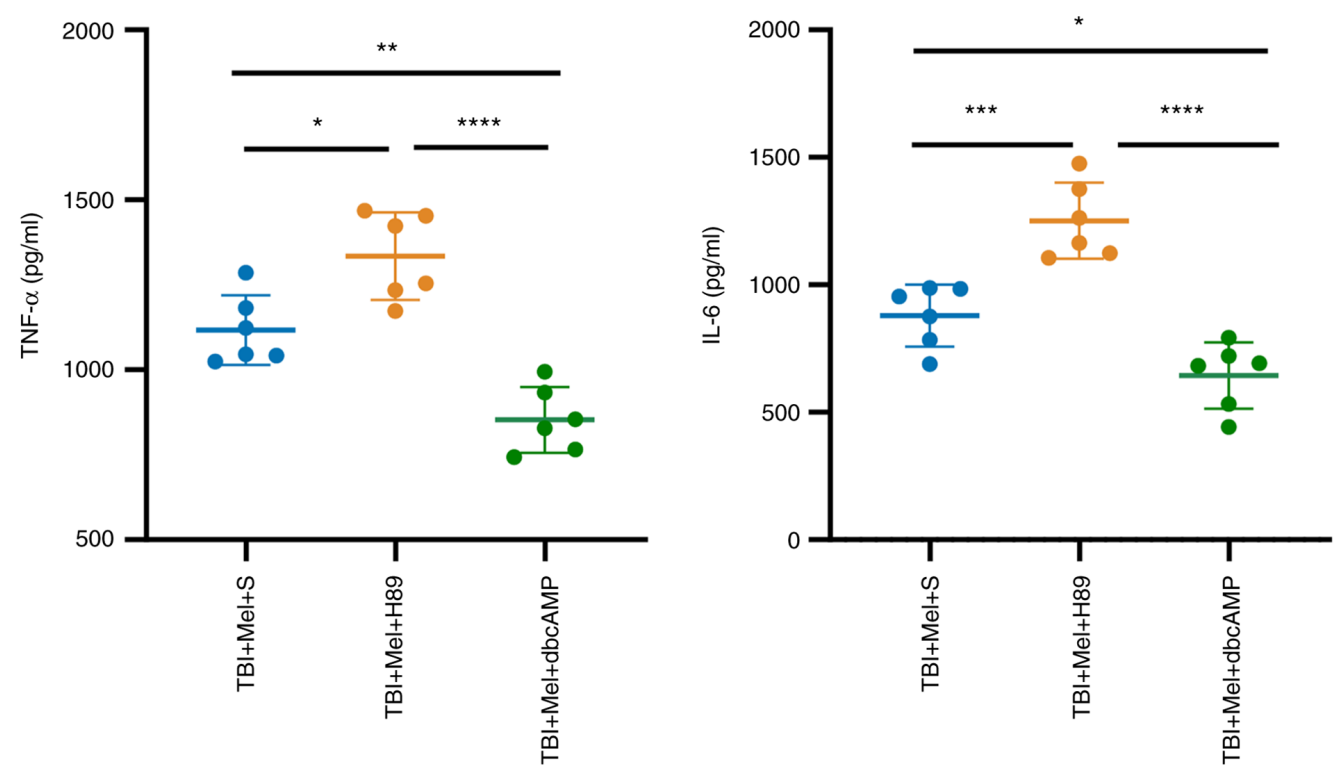

Figure 6. Effects of the administration of H89 and dbcAMP on the inflammatory cytokine expression of melatonin-treated TBI rats. Expression levels of TNF- $\alpha$ and IL-6 in the amygdala of rats. $\mathrm{n}=6$ rats per group. ${ }^{*} \mathrm{P}<0.05,{ }^{* *} \mathrm{P}<0.01,{ }^{* * * *} \mathrm{P}<0.001,{ }^{* * * * *} \mathrm{P}<0.0001$. TBI, traumatic brain injury; Mel, melatonin; $\mathrm{S}$, saline; dbcAMP, dibutyryl-cAMP.
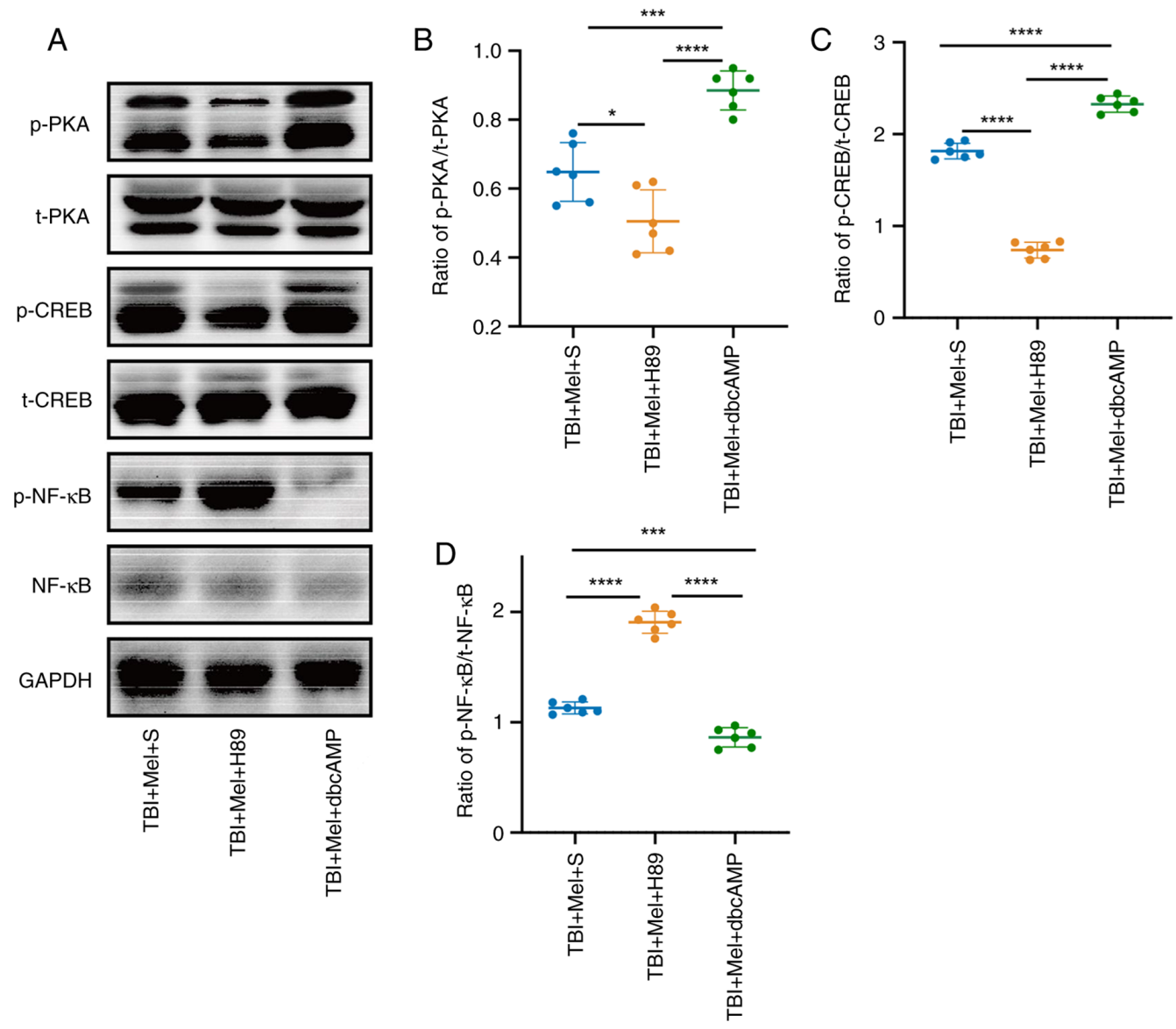

Figure 7. Melatonin alleviates TBI-induced anxiety-like behaviors through the PKA/CREB signaling pathway. (A) Representative results of immunoblotting of PKA, p-PKA, CREB, p-CREB, NF- $\mathrm{BB}$ and p-NF- $\mathrm{kB}$ in the amygdala tissue. Relative protein expression levels of (B) p-PKA/t-PKA, (C) p-CREB/t-CREB and

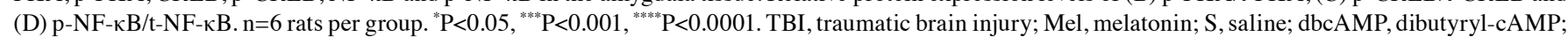
PKA, protein kinase A; CREB, cAMP-response element-binding protein; $\mathrm{p}-$-, phosphorylated; $\mathrm{t}-$, total.

Furthermore, the results revealed that $\mathrm{H} 89$ pretreatment before TBI in the TBI + Mel + H89 group induced significant decreases in the levels of $\mathrm{p}-\mathrm{PKA}(\mathrm{P}<0.05$ vs. $\mathrm{TBI}+\mathrm{Mel}+\mathrm{S}$; Fig. $7 \mathrm{~A}$ and $\mathrm{B})$ and $\mathrm{p}-\mathrm{CREB}(\mathrm{P}<0.0001$ vs. TBI $+\mathrm{Mel}+\mathrm{S}$; 
Fig. 7A and $\mathrm{C}$ ) in the amygdala compared with those in the $\mathrm{TBI}+\mathrm{Mel}+\mathrm{S}$ group. It was also revealed that the expression levels of $\mathrm{p}-\mathrm{NF}-\kappa \mathrm{B}$ were significantly increased in the TBI + $\mathrm{Mel}+\mathrm{H} 89$ group compared with those in the $\mathrm{TBI}+\mathrm{Mel}+\mathrm{S}$ group $(\mathrm{P}<0.0001$ vs. TBI $+\mathrm{Mel}+\mathrm{S}$; Fig. 7A and D). Although the expression levels of $\mathrm{p}-\mathrm{PKA}$ and $\mathrm{p}$-CREB were significantly elevated $(\mathrm{P}<0.001)$, the expression level of $\mathrm{p}-\mathrm{NF}-\kappa \mathrm{B}$ significantly declined in the TBI $+\mathrm{Mel}+$ dbcAMP group compared with that in the TBI $+\mathrm{Mel}+\mathrm{S}$ and $\mathrm{TBI}+\mathrm{Mel}+\mathrm{H} 89$ groups $(\mathrm{P}<0.001$; Fig. 7A-D). These findings revealed that melatonin could activate the PKA/CREB signaling pathway, whereas PKA inhibitors could partially reverse the neuroprotective effects of melatonin, which further aggravated the anxiety-like behavior and the release of inflammatory cytokines compared with those in the $\mathrm{TBI}+\mathrm{Mel}+\mathrm{S}$ group.

\section{Discussion}

TBI, represented by acute neurological injury, is considered to be a trigger for chronic traumatic disorders, including Alzheimer's disease and Parkinson's disease. However, no drug is currently available to effectively prevent these complications (30-32). Although previous studies have reported the involvement of anti-inflammation in the neuroprotective effects of melatonin $(25,33)$, the underlying molecular mechanisms remain unclear. The present study demonstrated the following primary novel findings: i) Melatonin decreased neuronal apoptosis in the amygdala post-TBI; ii) melatonin alleviated anxiety-like behaviors post-TBI; and iii) inhibition of p-PKA using $\mathrm{H} 89$ revealed that the protective effects of melatonin could be partially reversed post-TBI. To the best of our knowledge, the present study is the first to support the significance and important functions of PKA inhibition in the development of anxiety-like behaviors after TBI exposure to date.

TBI is likely linked to long-term emotional disorders such as depression and anxiety (34). A previous report indicated that in the developing brain, mild-to-moderate TBI results in early behavioral and metabolomics changes (35). The present study revealed that TBI caused anxiety-like behaviors in rats. The results demonstrated that the amount of rearing and number of squared crossed, as well as the average speed and total moving distance, were significantly decreased in the OFT. Moreover, there was a significant increase in the amount of time spent stationary in corners. Melatonin administration could relieve anxiety-like behaviors in rodents, but the protective effects of melatonin could be partially eliminated after the administration of PKA inhibitors. The amygdala is part of the limbic system of brain tissue that produces, recognizes and regulates emotions (36). The amygdala is prominently involved in a neurobiological model of anxiety (37). Therefore, the present study selected the amygdala as the focus of investigation in the primary research.

Recently, there is growing evidence indicating that melatonin is a potential antioxidant considered as a protectant against TBI (15). Due to this beneficial role, the present study explored the underlying mechanism by which melatonin protects the brain. The dose of melatonin $(10 \mathrm{mg} / \mathrm{kg}$, i.p) in the present study was based on a previous study (10), as well as our preliminary experiments. Intervention should be initiated as soon as possible after TBI, preferably within $4 \mathrm{~h}$ after injury, to achieve maximum neuroprotective effects (38); therefore, the time point of melatonin treatment used in the present study was $0 \mathrm{~h}$ post-TBI.

Astrocytes are the most abundant glial cells in the CNS and are traditionally considered as the only structural elements supporting the structure of the brain (39). However, the importance of astrocytes in the survival of neurons has been proposed in recent years $(40,41)$. In addition, drastically increased GFAP and allograft inflammatory factor 1 expression levels have been reported in the WD model of TBI (42). Astrocytes respond to TBI by activating the NF- $\kappa$ B pathway, which can result in the secretion of cytokines (43). For example, a study has demonstrated that reactive astrocytes may intensify inflammatory processes, if astrocytes exposed to an inflammatory stimulus produce proinflammatory cytokines such as IL-1 $\beta$ and TNF- $\alpha$ (44). IL-6-knockout can significantly reduce the neurological damage after TBI (45). Moreover, inhibition of TNF- $\alpha$ signaling can decrease neuronal apoptosis (46).

There are two main sources of inflammatory cytokines: One is the secretion of neutrophils and macrophages in the peripheral circulatory system, and the other is the microglia, astrocytes, oligodendrocytes and damaged neurons from the CNS. The present study aimed to explore the effects of melatonin on anxiety-like behaviors, neuronal apoptosis and astrocyte activation induced by TBI in rats. It was hypothesized that astrocyte activation might release more inflammatory cytokines, so the expression levels of TNF- $\alpha$ and IL-6 in cerebral tissues were tested. The present study revealed that astrocyte activation was increased in rats with TBI accompanied by increased neuronal apoptosis; moreover, after melatonin administration, the numbers of apoptotic neurons and activated astrocytes post-TBI were decreased. Furthermore, melatonin alleviated the anxiety-like behaviors induced by TBI. Therefore, it was hypothesized that the improvement of emotional changes induced by melatonin was associated with reduction of neuronal apoptosis and astrocyte activation.

Several previous studies have reported that the PKA/CREB signaling pathway is involved in apoptosis and correlates with cognitive function $(47,48)$. Activation of PKA/CREB signaling plays an important role in reducing neuronal death or neurotoxicity (21). Another previous study suggested that CREB activation also decreases the apoptosis of neuroblastoma cells (49). The present study demonstrated that pretreatment with H89, a special inhibitor of PKA, significantly decreased the expression levels of p-PKA and CREB, increased the expression levels of IL- 6 and TNF- $\alpha$ and reversed the improvement of anxiety-like behaviors induced by melatonin. However, pretreatment with dbcAMP, a PKA activator, further improved the anxiety-like behavior of rats. Overall, these results indicated that melatonin could alleviate the neuronal damage induced by TBI through the PKA/CREB signaling pathway. However, cAMP acts directly on three main effectors: PKA, protein directly activated by c-AMP (EPAC) and cyclic nucleotide-gated channels (50). In the hippocampus, EPAC contributes to the control of neuronal growth and differentiation and has been implicated in memory and learning as well as in anxiety and depression (51). Therefore, the role of EPAC in the findings in the present study cannot be ruled out.

The present study had several limitations. The experiments were limited only to the amygdala region, and changes 
in the ventromedial prefrontal cortex and hippocampus remain unexplored. The specific pathway of apoptosis, such as the expression of Bcl-2 and Bak, should be investigated in future experiments. CREB is a downstream factor of PKA, and the phosphorylation of PKA will directly affect the activation and phosphorylation of CREB. Lack of CREB inhibitor or knockdown experiments is another limitation of the present study.

To summarize, melatonin could alleviate TBI-induced anxiety-like behaviors in rats, and the underlying mechanism may be associated with activation of the PKA/CREB signaling pathway.

\section{Acknowledgements}

Not applicable.

\section{Funding}

The present study was supported by the Medical Science Research Project of Hebei Province (grant no. 20211745).

\section{Availability of data and materials}

All data generated or analyzed during this study are included in this published article.

\section{Authors' contributions}

LLX and ZZL performed the experiments and drafted the manuscript. SSL, YJF and MMQ helped to conduct the design of study and acquired the data. ZZL performed the statistical analyses. LLX conceived the study and revised the manuscript. MMQ and ZZL confirm the authenticity of all the raw data. All authors have read and approved the final manuscript.

\section{Ethics approval and consent to participate}

The present study was approved by the Animal Review Board of Cangzhou Central Hospital [Cangzhou, China; approval no. 2020-013-02(Z)]). All animal experiments were performed according to the standards of the Institutional Animal Care and Use Committee at the Cangzhou Central Hospital.

\section{Patient consent for publication}

Not applicable.

\section{Competing interests}

The authors declare that they have no competing interests.

\section{References}

1. Studlack PE, Keledjian K, Farooq T, Akintola T, Gerzanich V, Simard JM and Keller A: Blast-induced brain injury in rats leads to transient vestibulomotor deficits and persistent orofacial pain. Brain Inj 32: 1866-1878, 2018.

2. Capizzi A, Woo J and Verduzco-Gutierrez M: Traumatic brain injury: An overview of epidemiology, pathophysiology, and medical management. Med Clin North Am 104: 213-238, 2020.
3. Yatsiv I, Grigoriadis N, Simeonidou C, Stahel PF, Schmidt OI, Alexandrovitch AG, Tsenter J and Shohami E: Erythropoietin is neuroprotective, improves functional recovery, and reduces neuronal apoptosis and inflammation in a rodent model of experimental closed head injury. FASEB J 19: 1701-1703, 2005.

4. Adibhatla RM and Hatcher JF: Lipid oxidation and peroxidation in CNS health and disease: From molecular mechanisms to therapeutic opportunities. Antioxid Redox Signal 12: 125-169, 2010.

5. Adhikari A, Lerner TN, Finkelstein J, Pak S, Jennings JH, Davidson TJ, Ferenczi E, Gunaydin LA, Mirzabekov JJ, Ye L, et al: Basomedial amygdala mediates top-down control of anxiety and fear. Nature 527: 179-185, 2015.

6. Jayakar R, Tone EB, Crosson B, Turner JA, Anderson PL, Phan KL and Klumpp H: Amygdala volume and social anxiety symptom severity: Does segmentation technique matter? Psychiatry Res Neuroimaging 295: 111006, 2020.

7. Ji J, Kline AE, Amoscato A, Samhan-Arias AK, Sparvero LJ, Tyurin VA, Tyurina YY, Fink B, Manole MD, Puccio AM, et al: Lipidomics identifies cardiolipin oxidation as a mitochondrial target for redox therapy of brain injury. Nat Neurosci 15: 1407-1413, 2012

8. Hill RL, Singh IN, Wang JA, Kulbe JR and Hall ED: Protective effects of phenelzine administration on synaptic and non-synaptic cortical mitochondrial function and lipid peroxidation-mediated oxidative damage following TBI in young adult male rats. Exp Neurol 2020: 113322, 2020.

9. Chiu CC, Liao YE, Yang LY, Wang JY, Tweedie D, Karnati HK, Greig NH and Wang JY: Neuroinflammation in animal models of traumatic brain injury. J Neurosci Methods 272: 38-49, 2016.

10. Maas AI, Roozenbeek B and Manley GT: Clinical trials in traumatic brain injury: Past experience and current developments. Neurotherapeutics 7: 115-126, 2010.

11. Koh PO: Melatonin regulates the calcium-buffering proteins, parvalbumin and hippocalcin, in ischemic brain injury. J Pineal Res 53: 358-365, 2012.

12. Ding K, Wang H, Xu J, Li T, Zhang L, Ding Y, Zhu L, He J and Zhou M: Melatonin stimulates antioxidant enzymes and reduces oxidative stress in experimental traumatic brain injury: The Nrf2-ARE signaling pathway as a potential mechanism. Free Radic Biol Med 73: 1-11, 2014.

13. Wang Z, Ma C, Meng CJ, Zhu GQ, Sun XB, Huo L, Zhang J, Liu HX, He WC, Shen XM, et al: Melatonin activates the Nrf2-ARE pathway when it protects against early brain injury in a subarachnoid hemorrhage model. J Pineal Res 53: 129-137, 2012.

14. Osier N, McGreevy E, Pham L, Puccio A, Ren D, Conley YP, Alexander S and Dixon CE: Melatonin as a therapy for traumatic brain injury: A review of published evidence. Int J Mol Sci 19: $1539,2018$.

15. Rehman SU, Ikram M, Ullah N, Alam SI, Park HY, Badshah H, Choe K and Kim MO: Neurological Enhancement effects of melatonin against brain injury-induced oxidative stress, neuroinflammation, and neurodegeneration via AMPK/CREB signaling. Cells 8: 760, 2019.

16. Cheung RT, Tipoe GL, Tam S, Ma ES, Zou LY and Chan PS: Preclinical evaluation of pharmacokinetics and safety of melatonin in propylene glycol for intravenous administration. J Pineal Res 41: 337-343, 2006.

17. Kaur C, Sivakumar V, Robinson R, Foulds WS, Luu CD and Ling EA: Neuroprotective effect of melatonin against hypoxia-induced retinal ganglion cell death in neonatal rats. J Pineal Res 54: 190-206, 2013.

18. Zyuz'kov GN, Miroshnichenko LA, Polyakova TY, Stavrova LA, Simanina EV, Agafonov VI and Zhdanov VV: Participation of cAMP/PKA-Mediated signaling pathways in functional activity of regeneration-competent cells in the nervous tissue under conditions of ethanol-induced neurodegeneration. Bull Exp Biol Med 167: 723-727, 2019.

19. Gao X, Zhang X, Cui L, Chen R, Zhang C, Xue J, Zhang L, He W, Li J, Wei S, et al: Ginsenoside Rb1 promotes motor functional recovery and axonal regeneration in post-stroke mice through cAMP/PKA/CREB signaling pathway. Brain Res Bull 154: 51-60, 2020.

20. Ye J, Yin Y, Liu H, Fang L, Tao X, Wei L, Zuo Y, Yin Y, Ke D and Wang JZ: Tau inhibits PKA by nuclear proteasome-dependent PKAR $2 \alpha$ elevation with suppressed CREB/GluA1 phosphorylation. Aging Cell 19: e13055, 2020. 
21. Ma CL, Li L, Yang GM, Zhang ZB, Zhao YN, Zeng XF, Zhang DX, Yu Y, Shi ZJ, Yan QW, et al: Neuroprotective effect of gastrodin in methamphetamine-induced apoptosis through regulating cAMP/PKA/CREB pathway in cortical neuron. Hum Exp Toxicol 39: 1118-1129, 2020.

22. Yang L, Shi LJ, Yu J and Zhang YQ: Activation of protein kinase $\mathrm{A}$ in the amygdala modulates anxiety-like behaviors in social defeat exposed mice. Mol Brain 9: 3, 2016.

23. Sung JY, Bae JH, Lee JH, Kim YN and Kim DK: The melatonin signaling pathway in a long-term memory in vitro study. Molecules 23: 737, 2018.

24. Li C, Chen T, Zhou H, Feng Y, Hoi MPM, Ma D, Zhao C, Zheng Y and Lee SMY: BHDPC is a novel neuroprotectant that provides anti-neuroinflammatory and neuroprotective effects by inactivating NF- $\kappa \mathrm{B}$ and activating PKA/CREB. Front Pharmacol 25: 614,2018

25. Ding K, Xu J, Wang H, Zhang L, Wu Y and Li T: Melatonin protects the brain from apoptosis by enhancement of autophagy after traumatic brain injury in mice. Neurochem Int 91: 46-54, 2015.

26. Song J, Cheon SY, Lee WT, Park KA and Lee JE: PKA Inhibitor H89 (N-[2-p-bromocinnamylamino-ethyl]-5isoquinolinesulfonamide) attenuates synaptic dysfunction and neuronal cell death following ischemic injury. Neural Plast 2015: $374520,2015$.

27. Salehi F, Hosseini-Zare MS, Aghajani H, Seyedi SY, Hosseini-Zare MS and Sharifzadeh M: Effect of bucladesine, pentoxifylline, and $\mathrm{H}-89$ as cyclic adenosine monophosphate analog, phosphodiesterase, and protein kinase A inhibitor on acute pain. Fundam Clin Pharmacol 31: 411-419, 2017

28. Shishido H, Ueno M, Sato K, Matsumura M, Toyota Y, Kirino Y, Tamiya T, Kawai N and Kishimoto Y: Traumatic brain injury by weight-drop method causes transient amyloid- $\beta$ deposition and acute cognitive deficits in mice. Behav Neurol 2019: 3248519, 2019.

29. Oh HM, Lee JS, Kim SW, Oh YT, Kim WY, Lee SB, Cho YR, Jeon YJ, Cho JH and Son CG: Uwhangchungsimwon, A standardized herbal drug, exerts an anti-depressive effect in a social isolation stress-induced mouse model. Front Pharmacol 10: 1674, 2020.

30. Kondo A, Shahpasand K, Mannix R, Qiu J, Moncaster J, Chen CH, Yao Y, Lin YM, Driver JA, Sun Y, et al: Antibody against early driver of neurodegeneration cis P-tau blocks brain injury and tauopathy. Nature 523: 431-436, 2015.

31. Tajiri N, Acosta SA, Shahaduzzaman M, Ishikawa H, Shinozuka K, Pabon M, Hernandez-Ontiveros D, Kim DW, Metcalf C, Staples M, et al: Intravenous transplants of human adipose-derived stem cell protect the brain from traumatic brain injury-induced neurodegeneration and motor and cognitive impairments: Cell graft biodistribution and soluble factors in young and aged rats. J Neurosci 34: 313-326, 2014

32. Yu S, Kaneko Y, Bae E, Stahl E, Wang Y, van Loveren H, Sanberg PR and Borlongan CV: Severity of controlled cortical impact traumatic brain injury in rats and mice dictates degree of behavioral deficits. Brain Res 1287: 157-163, 2009.

33. Chen H, Sun X, Yang X, Hou Y, Yu X, Wang Y, Wu J, Liu D, Wang $\mathrm{H}$, Yu J and Yi W: Dexmedetomidine reduces ventilator-induced lung injury (VILI) by inhibiting Toll-like receptor 4 (TLR4)/nuclear factor (NF)- $\mathrm{B}$ signaling pathway. Bosn J Basic Med Sci 18: 162-169, 2018.

34. Popovitz J, Mysore SP and Adwanikar H: Long-term effects of traumatic brain injury on anxiety-like behaviors in mice: Behavioral and neural correlates. Front Behav Neurosci 13: 6 , 2019.

35. Chitturi J, Li Y, Santhakumar V and Kannurpatti SS: Early behavioral and metabolomic change after mild to moderate traumatic brain injury in the developing brain. Neurochem Int 120 75-86, 2018

36. Šimić G, Tkalčić M, Vukić V, Mulc D, Španić E, Šagud M, Olucha-Bordonau FE, Vukšić M and R Hof P: Understanding emotions: Origins and roles of the amygdala. Biomolecules 11: $823,2021$.
37. Herrington JD, Miller JS, Pandey J and Schultz RT: Anxiety and social deficits have distinct relationships with amygdala function in autism spectrum disorder. Soc Cogn Affect Neurosci 11: 907-914, 2016.

38. Sullivan PG, Sebastian AH and Hall ED: Therapeutic window analysis of the neuroprotective effects of cyclosporine A after traumatic brain injury. J Neurotrauma 28: 311-318, 2011.

39. Neal M and Richardson JR: Epigenetic regulation of astrocyte function in neuroinflammation and neurodegeneration. Biochim Biophys Acta Mol Basis Dis 1864: 432-443, 2018.

40. Chávez CE, Oyarzún JE, Avendaño BC, Mellado LA, Inostroza CA, Alvear TF and Orellana JA: The opening of connexin 43 hemichannels alters hippocampal astrocyte function and neuronal survival in prenatally LPS-Exposed adult offspring. Front Cell Neurosci 13: 460, 2019.

41. Fujita A, Yamaguchi H, Yamasaki R, Cui Y, Matsuoka Y, Yamada KI and Kira JI: Connexin 30 deficiency attenuates A2 astrocyte responses and induces severe neurodegeneration in a 1-methyl-4-phenyl-1,2,3,6-tetrahydropyridine hydrochloride Parkinson's disease animal model. J Neuroinflammation 15: 227 , 2018.

42. Witcher KG, Bray CE, Dziabis JE, McKim DB, Benner BN, Rowe RK, Kokiko-Cochran ON, Popovich PG, Lifshitz J, Eiferman DS and Godbout JP: Traumatic brain injury-induced neuronal damage in the somatosensory cortex causes formation of rod-shaped microglia that promote astrogliosis and persistent neuroinflammation. Glia 66: 2719-2736, 2018.

43. Gao TL, Yuan XT, Yang D, Dai HL, Wang WJ, Peng X, Shao HJ, Jin ZF and Fu ZJ: Expression of HMGB1 and RAGE in rat and human brains after traumatic brain injury. J Trauma Acute Care Surg 72: 643-649, 2012

44. Hirsch EC, Breidert T, Rousselet E, Hunot S, Hartmann A and Michel PP: The role of glial reaction and inflammation in Parkinson's disease. Ann N Y Acad Sci 991: 214-228, 2003.

45. Ley EJ, Clond MA, Singer MB, Shouhed D and Salim A: IL6 deficiency affects function after traumatic brain injury. J Surg Res 170: 253-256, 2011

46. Baratz R, Tweedie D, Wang JY, Rubovitch V, Luo W, Hoffer BJ, Greig NH and Pick CG: Transiently lowering tumor necrosis factor-alpha synthesis ameliorates neuronal cell loss and cognitive impairments induced by minimal traumatic brain injury in mice. J Neuroinflammation 12: 45, 2015.

47. Xing J, Han D, Xu D, Li X and Sun L: CREB Protects against temporal lobe epilepsy associated with cognitive impairment by controlling oxidative neuronal damage. Neurodegener Dis 19: 225-237, 2019

48. Yang Y, Ma S, Wei F, Liang G, Yang X, Huang Y, Wang J and Zou Y: Pivotal role of cAMP-PKA-CREB signaling pathway in manganese-induced neurotoxicity in PC12 cells. Environ Toxicol 34: 1052-1062, 2019.

49. Ciani E, Guidi S, Della Valle G, Perini G, Bartesaghi R and Contestabile A: Withdrawal: Nitric oxide protects neuroblastoma cells from apoptosis induced by serum deprivation through cAMP-response element-binding protein (CREB) activation. J Biol Chem 295: 3391, 2020

50. Purves GI, Kamishima T, Davies LM, Quayle JM and Dart C: Exchange protein activated by cAMP(Epac)mediatescAMP-dependent but protein kinase A-insensitive modulation of vascular ATP-sensitive potassium channels. J Physiol 587: 3639-3650, 2009.

51. Aesoy R, Muwonge H, Asrud KS, Sabir M, Witsoe SL, Bjornstad R, Kopperud RK, Hoivik EA, Doskeland SO and Bakke M: Deletion of exchange proteins directly activated by cAMP (Epac) causes defects in hippocampal signaling in female mice. PLoS One 13: e0200935, 2018.

This work is licensed under a Creative Commons Attribution-NonCommercial-NoDerivatives 4.0 International (CC BY-NC-ND 4.0) License. 